\title{
Volumetry of Mesiotemporal Structures Reflects Serostatus in Patients with Limbic Encephalitis
}

\author{
(D) Er. Ernst, (D) B. David, (D). Gaubatz, (DI. Domínguez-Narciso, (D) Lüchters, (D)A.J. Becker, (D)B. Weber, (D) E. Hattingen,
} (D) C.E. Elger, and DT. Rüber

\begin{abstract}
BACKGROUND AND PURPOSE: Limbic encephalitis is an autoimmune disease. A variety of autoantibodies have been associated with different subtypes of limbic encephalitis, whereas its MR imaging signature is uniformly characterized by mesiotemporal abnormalities across subtypes. Here, we hypothesized that patients with limbic encephalitis would show subtype-specific mesiotemporal structural correlates, which could be classified by supervised machine learning on an individual level.
\end{abstract}

MATERIALS AND METHODS: TIWI MPRAGE scans from 46 patients with antibodies against glutamic acid decarboxylase and 34 patients with antibodies against the voltage-gated potassium channel complex (including 10 patients with leucine-rich glioma-inactivated 1 autoantibodies) and 48 healthy controls were retrospectively ascertained. Parcellation of the amygdala, hippocampus, and hippocampal subfields was performed using FreeSurfer. Volumes were extracted and compared between groups using unpaired, 2-tailed $t$ tests. The volumes of hippocampal subfields were analyzed using a multivariate linear model and a binary decision tree classifier.

RESULTS: Temporomesial volume alterations were most pronounced in an early stage and in the affected hemispheric side of patients. Statistical analysis revealed antibody-specific hippocampal fingerprints with a higher volume of CA1 in patients with glutamic acid decarboxylase-associated limbic encephalitis $(P=.02)$, compared with controls, whereas CA1 did not differ from that in controls in patients with voltage-gated potassium channel complex autoantibodies. The classifier could successfully distinguish between patients with autoantibodies against leucine-rich glioma-inactivated 1 and glutamic acid decarboxylase with a specificity of $87 \%$ and a sensitivity of $80 \%$.

CONCLUSIONS: Our results suggest stage-, side- and antibody-specific structural correlates of limbic encephalitis; thus, they create a perspective toward an MR imaging-based diagnosis.

ABBREVIATIONS: CASPR2 = contactin-associated proteinlike 2; EEG = electroencephalogram; GAD = glutamic acid decarboxylase; GAD-LE = limbic en cephalitis with glutamic acid decarboxylase-associated autoantibodies; LE = limbic encephalitis; LGII = leucine-rich glioma-inactivated 1; VGKC = voltagegated potassium channel complex; VGKC-LE = limbic encephalitis with voltage-gated potassium channel complex-associated autoantibodies

imbic encephalitis (LE) is an autoimmune disease defined by subacute short-term memory loss and psychiatric

Received June 21, 2019; accepted after revision September 11.

From the Department of Epileptology (L.E., B.D., J.G., I.D.-N., C.E.E., T.R.), Department of Neuropathology (A.J.B.), Institute for Experimental Epileptology and Cognition Research (B.W.), and Department of Radiology (E.H.), University of Bonn Medical Center, Bonn, Germany; Center for Development Research (G.L.), University of Bonn, Bonn, Germany; Department of Neuroradiology (E.H.), Goethe University Frankfurt, Frankfurt, Germany; and Epilepsy Center Frankfurt Rhine-Main (T.R.), Department of Neurology, and Center for Personalized Translational Epilepsy Research (T.R.), Goethe-University Frankfurt, Frankfurt am Main, Germany Leon Ernst received support from the Promotionskolleg Neuroimmunology of the University of Bonn and the Else-Kröner-Fresenius Stiftung. Christian E. Elger received grants from Deutsche Forschungsgemeinschaft, Bundesministerium für Bildung und Forschung, and Marga and Walter Boll Stiftung. Theodor Rüber is supported by the BONFOR research commission of the medical faculty of the University of Bonn.

Paper previously presented, in part, at: Annual Meeting of the German Society for Neurology, November 1-3, 2018, Berlin, Germany; the BonnBrain Conference, March 25-27, 2019, Bonn, Germany; and Annual Meeting of the Organization for Human Brain Mapping, June 9-13, 2019; Rome, Italy. abnormalities often involving temporal lobe epilepsy. ${ }^{1-3}$ Several autoantibodies have been associated with different subtypes of LE, sharing clinical core features like the limbic syndrome, and may be discriminated by other characteristics such as comorbidities and response to immunotherapy. ${ }^{4,5}$ Autoantibodies against the voltage-gated potassium channel complex (VGKC) and glutamic acid decarboxylase (GAD) are commonly found in patients with LE with temporal lobe epilepsy. ${ }^{6}$ Whereas patients with LE with autoantibodies against GAD and VGKC (GAD-LE and VGKC-LE) all display an early and prominent development of

Please address correspondence to Theodor Rüber, MD, Department of Epileptology, University of Bonn Medical Center, Sigmund-Freud Str 25, 53127 Bonn, Germany; e-mail: theodor.rueber@ukbonn.de

--.- Indicates open access to non-subscribers at www.ajnr.org

$\equiv$ Indicates article with supplemental on-line tables.

http://dx.doi.org/10.3174/ajnr.A6289 
epileptic seizures, those with VGKC-LE are more responsive to immunotherapy. Recently, 2 antigenic components of the VGKC complex that are targeted by different autoantibodies have been identified: the leucine-rich glioma-inactivated 1 (LGI1) and contactin-associated proteinlike 2 (CASPR2). ${ }^{7,8}$ However, 12\%-33\% of those with VGKC-LE are negative for both of these subantigens. ${ }^{8,9}$ Furthermore, the detection of specific autoantibodies may not exclude the presence of other pathologic neuronal autoantibodies yet undiscovered. Therefore, it has been suggested that the diagnosis of LE should rely less on autoantibodies and more on clinical examinations and MR imaging., ${ }^{3,10}$

MR imaging is especially relevant when the results of antibody testing are not yet available, and follow-up scans may be helpful for assessing the response to therapy. However, MR imaging findings correlate imperfectly with the course of disease, and antibody-specific MR imaging signatures have not yet been found on an individual level, though antibody-specific imaging features of LE are increasingly recognized on a group level. ${ }^{11-14}$ Thus, neuroimaging research in patients with LE may not only increase our pathophysiologic understanding of this disease but also help to define imaging biomarkers needed on clinical grounds. By harnessing modern techniques for hippocampal subfield segmentation, we sought to bridge the gap between clinical and radiologic findings. Here, we hypothesized that patients with LE would show stage-specific, side-specific, and antibody-specific mesiotemporal structure correlates, which could be classified by supervised machine learning on an individual level.

\section{MATERIALS AND METHODS Subjects}

We retrospectively ascertained clinical and $\mathrm{MR}$ imaging data from patients with LE who were treated the Department of Epileptology at University of Bonn Medical Center between April 2006 and June 2017 and met the following inclusion criteria: 1) 18 years of age or older, 2) having a diagnosis of a limbic syndrome (with at least 1 of the following symptoms: temporal lobe seizures, episodic memory disturbance, or psychiatric symptoms with affective disturbance), 3) serologically proved autoantibodies against the VGKC complex (or, if tests were available, against its antigenic components LGI1 or CASPR2) or GAD, and 4) availability of at least one 3D T1-weighted MPRAGE MR imaging scan. Bilateral mesiotemporal T2-FLAIR hyperintensities in routine clinical imaging ${ }^{3}$ were not considered an inclusion criterion, to avoid a selection bias of image analysis. Forty-six patients with GAD-LE and 34 patients with VGKC-LE were included in the study (80 patients in total, 36 men). Both GAD-LE and VGKCLE groups were divided into 2 subgroups, respectively, depending on the time point of the MR imaging acquisition: The early group included T1WI scans acquired $<24$ months after the onset of the first LE-related symptom and is referred to as the early GAD group (23 patients in total, 7 men; age at MR imaging, $34.2 \pm$ 11.2 years) or early VGKC group (25 patients in total, 16 men; age at MR imaging, $59.5 \pm 15.2$ years). The late group included the most recent available T1WI scans acquired at least 24 months after the onset of epilepsy and is referred to as late GAD group (33 patients in total, $10 \mathrm{men}$; age at MR imaging, $33.9 \pm 12.3$ years) or late VGKC group (22 patients in total, 10 men; age at
MR imaging, $57.0 \pm 16.8$ years). ${ }^{15}$ Ten patients from the GAD group and 13 patients from the VGKC group were included in both the early and the late groups because they were scanned repeatedly less than and more than 24 months after the onset of the first LE-related symptom.

Control subjects with no history of psychiatric or neurologic disorders were ascertained and individually matched with regard to sex, age at MR imaging, and MR imaging sequence (see "Image Acquisition"). This process resulted in a total of 4 control groups for the 4 patient groups: an early GAD group, late GAD group, early VGKC group, and late VGKC group (for a summary of patient and controls groups see Table 1; for details, see supporting information in On-line Tables 1 and 2). The study was approved by the local institutional review board, and all participants had provided written informed consent (as part of previous studies).

\section{Antibody Testing}

After 2014, screening for onconeuronal antibodies was performed using semiquantitative immunoblots coated with recombinant antigen or antigen fragments.

Before 2014, detection of GAD antibodies as well as of VGKC complex antibodies in serum was performed using a radioimmunoprecipitation assay. Antibodies against LGI1 and CASPR2 were detected by indirect immunofluorescence. These tests were not performed before 2010; thus, 13 patients in the early VGKC group and 14 patients in the late VGKC group have not been tested for CASPR2 or LGI1 (for details, see supporting information in On-line Appendix).

\section{Treatment}

Immunotherapy was conducted according to guidelines set forth by the German Society for Neurology and expert opinion. It included first-line steroids, plasma exchange, and intravenous immunoglobulins, as well as second-line azathioprine, mycophenolate mofetil, rituximab, basiliximab, and cyclophosphamide. ${ }^{2,16,17}$ Immunotherapy was administered in addition to antiepileptic medication (for a summary see Table 1; for details, see the supporting information in On-line Tables 1 and 2).

\section{Image Acquisition}

Imaging data were acquired on a 3T Magnetom Trio scanner (Siemens, Erlangen, Germany) using an 8-channel receive head coil. For each scan time point, we acquired a T1WI MPRAGE sequence with the following parameters: voxel size $=1.0 \times$ $1.0 \times 1.0 \mathrm{~mm}^{3}, \mathrm{TR}=1570 \mathrm{~ms}, \mathrm{TE}=3.42 \mathrm{~ms}$, flip angle $=15^{\circ}, \mathrm{ma}-$ trix $=256 \times 256$ pixel. Due to a scanner update at the beginning of 2014, T1WI scans after this time point were acquired using a 32-channel head coil and the following parameters: voxel size $=$ $0.8 \times 0.8 \times 0.8 \mathrm{~mm}^{3}, \mathrm{TR}=1660 \mathrm{~ms}, \mathrm{TE}=2.54 \mathrm{~ms}$, flip angle $=9^{\circ}$, matrix $=320 \times 320$ pixel. All control groups were matched with regard to sequence (see above).

\section{Image Analysis}

Volumetric analysis of the hippocampus, hippocampal subfields, amygdala, and intracranial volume was performed using the FreeSurfer, Version 6.0.0 image analysis suite (http://surfer.nmr. mgh.harvard.edu). ${ }^{18,19}$ The hippocampal substructures segmented by FreeSurfer, Version 6.0.0, included the following: 
Table 1: Demographic and clinical characteristics of subgroups of patients with limbic encephalitis and their corresponding control groups

\begin{tabular}{|c|c|c|c|c|}
\hline & GAD-LE & VGKC-LE & GAD-CON & VGKC-CON \\
\hline \multicolumn{5}{|l|}{ Early group } \\
\hline No. (men) & $23(7)$ & $25(16)$ & $23(7)$ & $25(16)$ \\
\hline Age at MR imaging (mean) (yr) & $34.2 \pm 11.2$ & $59.5 \pm 15.2$ & $35.4 \pm 10.6$ & $57.2 \pm 13.2$ \\
\hline Time between onset ${ }^{a}$ and scan (mean) (mo) & $9.5 \pm 7.4$ & $7.6 \pm 6.1$ & NA & NA \\
\hline Interictal EEG lateralization & $5 / 12 / 1 / 5$ & $7 / 8 / 2 / 8$ & NA & NA \\
\hline \multicolumn{5}{|l|}{ ( $n=$ right/left/bilateral/unclear) } \\
\hline No. of bilateral mesiotemporal FLAIR-T2-hyperintensities & 2 & 5 & NA & NA \\
\hline No. of first-line immunotherapies & 3 & 9 & NA & NA \\
\hline No. of second-line immunotherapies & 0 & 1 & NA & NA \\
\hline \multicolumn{5}{|l|}{ Late group } \\
\hline No. $(m e n)^{b}$ & $33(10)$ & $22(10)$ & $33(10)$ & $22(10)$ \\
\hline Age at MR imaging (mean) (yr) & $33.9 \pm 12.3$ & $57.0 \pm 16.8$ & $34.4 \pm 11.9$ & $53.2 \pm 13.2$ \\
\hline Time between onset ${ }^{\mathrm{a}}$ and scan (mean) (mo) & $62.5 \pm 26.2$ & $61.5 \pm 23.6$ & NA & NA \\
\hline $\begin{array}{l}\text { Interictal EEG lateralization } \\
\text { ( } n=\text { right/left/bilateral/unclear) }\end{array}$ & $5 / 11 / 3 / 12$ & $4 / 8 / 1 / 9$ & NA & NA \\
\hline No. of bilateral mesiotemporal FLAIR-T2-hyperintensities & 4 & 3 & NA & NA \\
\hline No. of first-line immunotherapies & 14 & 18 & NA & NA \\
\hline No. of second-line immunotherapies & 6 & 4 & NA & NA \\
\hline
\end{tabular}

Note:-NA indicates not applicable; CON, matched healthy control group.

${ }^{a}$ Onset of the first limbic encephalitis-related symptom.

${ }^{b}$ Patients scanned repeatedly were included in both (early and late) study groups (repeat scans GAD-LE: $n=10$; repeat scans VGKC-LE, $n=13$ )

CA1, CA2/3, CA4, fimbria, hippocampal tail, the hippocampusamygdala transition area, granule cell layer of the dentate gyrus, presubiculum, parasubiculum, subiculum, molecular layer, and hippocampal fissure. The volume of the hippocampal fissure does not contribute to computation of the whole hippocampal volume and was deliberately excluded from the analysis. ${ }^{20}$ Parcellation results were visually checked for accuracy and alignment by 2 independent raters (L.E. and I.D.-N.), supervised by an experienced neuroradiologist (E.H.). In case of inexact alignment, FreeSurfer parcellation was repeated with adjusted parameters. If parcellation results were again rated unsatisfactory by 1 or 2 raters, the subject was excluded. This procedure led to the exclusion of 1 subject from the VGKC group. All analyzed volumes were adjusted by the intracranial volume minus the ventricular volume. Patient-control differences in the hippocampal subfield volume were $Z$-transformed, color-coded, and visualized in a $3 \mathrm{D}$ model of a representative subject.

\section{Reorientation of Images}

For analysis, volumes of the amygdala, hippocampus, and hippocampal subfields were regrouped according to the lateralization in the interictal electroencephalogram (EEG), thus, allowing differential analyses of the affected and unaffected hemispheres to estimate the lateralization of pathologic structural alterations. To control for the effect of a left-right bias, we also regrouped an equal number of controls. If a clear focus could not be determined in the EEG or if a bilateral pathologic pattern was observed, we used the mean values from both hemispheres for statistical analysis.

\section{Statistical Analysis}

Statistical analysis was performed using STATA 2015, Stata Statistical Software, Release 14 (StataCorp, College Station, Texas). The volumetric data of the amygdala and the entire hippocampus were compared between groups using unpaired, 2tailed $t$ tests. The joint effect of group on all hippocampal subfields was tested by means of a multivariate linear model. Subsequently, post hoc tests were conducted, which were protected by the Fisher least-significant difference. ${ }^{21}$ Test results were considered statistically significant if $P<.05$. Visualization of analyses results was with Matlab's Statistics Toolbox (Release 2012b; MathWorks, Natick, Massachusetts).

\section{Supervised Machine Learning Classification}

We set up a binary decision tree classifier using the Scikit-Learn toolkit ${ }^{22}$ to distinguish early LE groups on the basis of the volumetry of the hippocampal subfields on the affected side. To prevent age-related brain atrophy (especially in patients with LGI1) from influencing classification results, we corrected all volumetry data for the intracranial volume of the respective subject. Only early LE groups were tested because structural between-group differences may be thought to be most clearcut at a pretherapeutic stage when potential future clinical applications are most needed. Decision trees are a type of nonparametric supervised statistical learning method. ${ }^{23}$ The generalizability of our decision tree was assessed using a leaveone-out cross-validation scheme. Hyperparameter tuning was performed using an exhaustive grid search over the best-split criteria, maximum tree depth, minimum number of samples required to split an internal node, minimum number of samples at a leaf node, and the maximum number of features considered at every split. The F1 score was used as an optimization metric. However, default parameters set by Scikit-Learn yielded the best estimator for our problem (please refer to Scikit-Learn API [https://keras.io/scikit-learn-api/] for a fullparameter description and default values). To statistically compare the accuracy of our classifier against chance level, we tested its performance against null distributions by randomly shuffling the group labels in our dataset. In this way, 100,000 unique permutations were tested. The Gini importance of classification features was assessed post hoc on the basis of the model fit. 


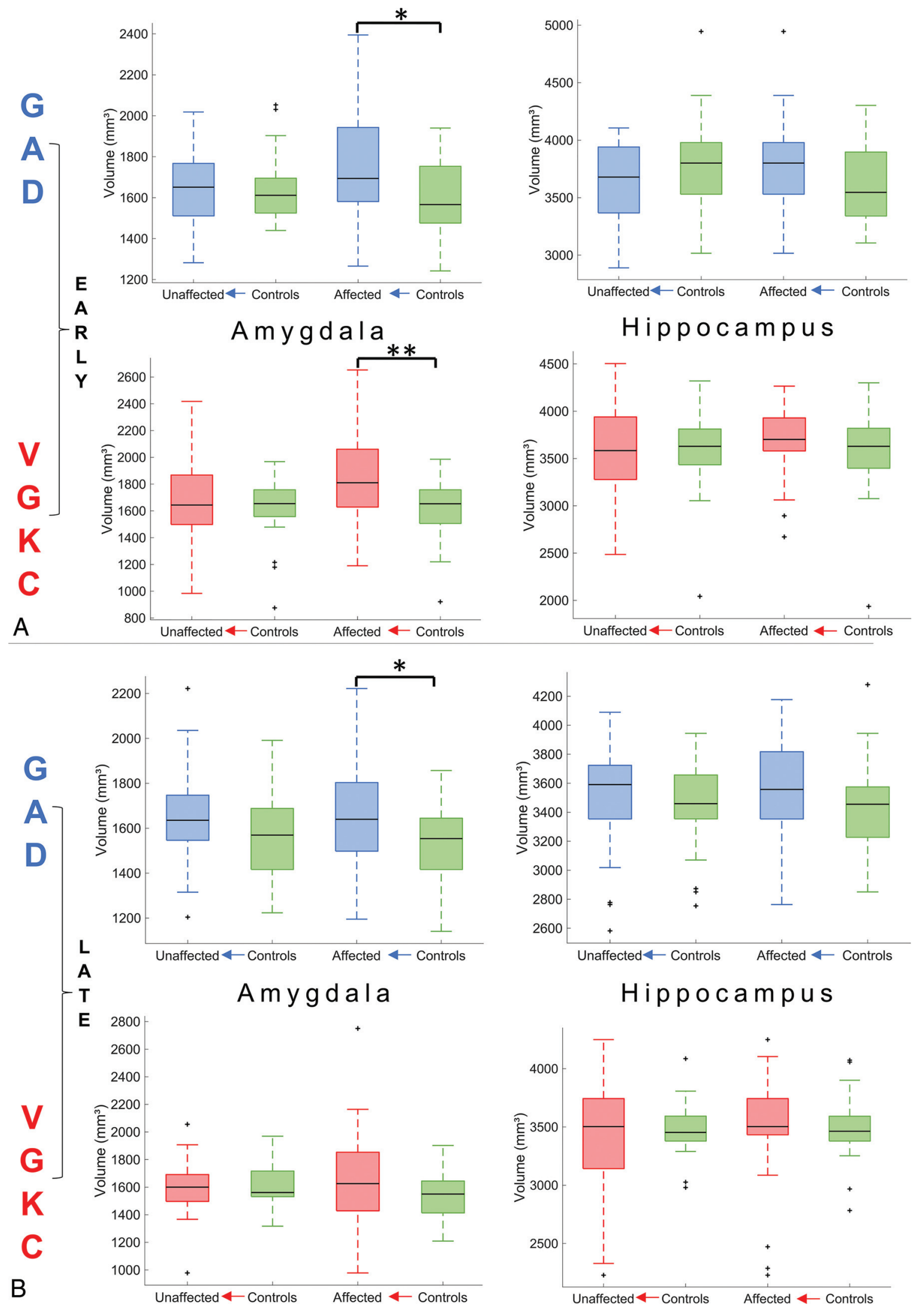

FIG 1. Volumetry of the amygdala and hippocampus. $A$, Volumetry in the early group. $B$, Volumetry in the late group. Asterisks represent levels of significance (single asterisk indicates .05; double asterisks, .01). 


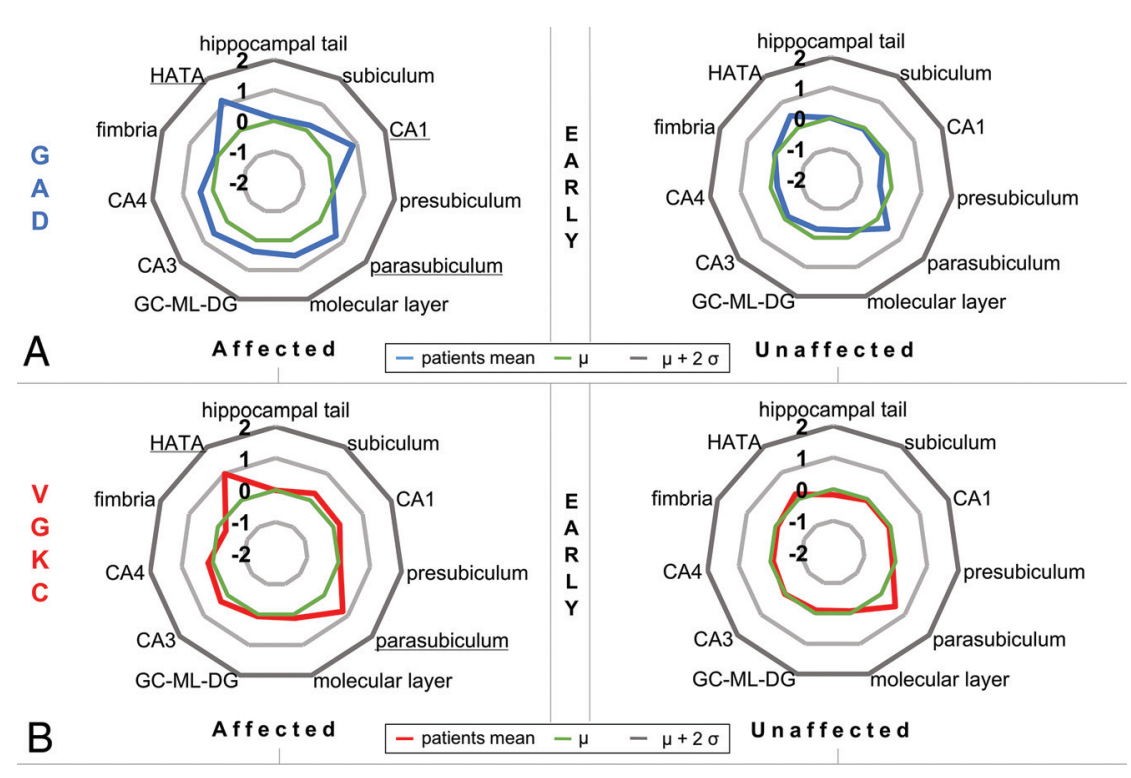

FIG 2. Starplots showing hippocampal subfield volume differences between patients in the early group and controls. $Z$ values reflect the hippocampal subfield volume difference between patients in the early group and controls in the affected and the unaffected hemispheric sides. Underlined words indicate statistical significance of the respective hippocampal subfield $(P<.05)$ in post hoc tests between patients and controls following a multivariate linear model (see MATERIALS AND METHODS and supporting information in On-line Tables 3-8). A, Relative hippocampal subfield volumes of the affected and unaffected sides of GAD-LE. B, Relative hippocampal subfield volumes of the affected and unaffected sides of VGKC-LE. GC-ML-DG indicates granule cell layer of the dentate gyrus; HATA, hippocampus-amygdala transition area.

\section{RESULTS}

\section{Volumetry of Amygdala and Hippocampus}

Compared with matched controls, the amygdala showed a higher volume in early GAD-LE and late GAD-LE (both, $P=.03$; Fig 1 ), whereas VGKC-LE showed only a higher volume in the early group $(P=.004)$. No group differences between early and late groups and controls were found in the volume of the hippocampus.

\section{Volumetry of Hippocampal Subfields}

Volumetry of hippocampal subfields yielded hippocampal fingerprints specific to the serogroup and hemispheric side. Multivariate linear models indicated statistically significant volume differences between patients and controls in the affected hemisphere for the early GAD group, the late GAD group, and the early VGKC group $(P<.02)$, but not for the late VGKC group $(P=.12)$. The most noticeable subfield differences were found among the early groups: We observed that volumes of CA1, the hippocampus-amygdala transition area, and the parasubiculum were significantly higher on the affected side in patients with early GAD-LE compared with their matched controls (all, $P<.04$ ), while in early VGKC-LE, this was the case for the hippocampus-amygdala transition area and the parasubiculum (all $P<.01$; see Fig 2 and supporting information in On-line Tables $3-8)$. Because 10 patients in the early VGKC group were positively tested for autoantibodies against LGI1, we also compared the patients with LGI1 and the early VGKC group without LGI1 with matched controls and found significant differences $(P<.01$, Fig 3). Please see the supporting information in On-line Tables
3-8 and On-line Tables 9 and 10 for the full results of the statistical analyses.

The anatomic 3D visualization of results illustrates subregional volume alterations. This and the schematic plots of $Z$ values as a function of the estimated anatomic distance from the amygdala reveal an anterior-to-posterior gradient with volume-increased hippocampal subfields in the anterior part (as opposed to the posterior part of the hippocampus) only in the early $\mathrm{GAD}$ group (Fig 4).

\section{Classification Performance}

The decision tree could successfully distinguish between patients with early LGI1 and early GAD with a specificity of $87 \%$ and sensitivity of $80 \%$ (positive predictive value $=72.2 \%$; negative predictive value $=90.9 \%$; accuracy $=84.8 \%$; F1 score $=0.76$; Fig 3). Permutation testing further confirmed an above-chance classification performance $(P=.002)$. A post hoc analysis of feature selection showed the fimbria to be of the highest importance for classification (Gini = 0.49 ), followed by CA1 ( Gini $=0.27$ ), the presubiculum (Gini $=0.13$ ), and the hippocampal tail $(\mathrm{Gini}=0.11)$. Differentiation between the early GAD group and the early VGKC group reached only a specificity of $59 \%$ and a sensitivity of $61 \%$ (positive predictive value = $64.1 \%$; negative predictive value $=56.5 \%$; accuracy $=60.4 \% ; \mathrm{F} 1$ score $=0.63$ ), and permutation testing could not confirm an above-chance classification performance $(P=.16)$.

\section{DISCUSSION}

This study yields 4 main results: Mesiotemporal volume alterations in patients with antibody-associated LE are most prominent in an early stage (stage-specificity), they primarily occur in the clinically affected hemispheric side (side-specificity), and hippocampal volume alterations are antibody-specific for GAD, VGKC, and LGI1 on a group level (autoantibody-specificity) and may discriminate between GAD and LGI1 on an individual level using a decision tree classifier (classification).

\section{Stage-Specificity}

Between those with GAD-LE and controls, volumetry of the amygdala yields differences in the early and the late groups. Volumetry of VGKC-LE and controls in contrast shows amygdala group differences only in the early group (Fig 1, with supporting information in On-line Table 9). The results of amygdala volumetry are paralleled by results of the multivariate linear model of hippocampal-subfield volumetry (see supporting information in On-line Tables 3-8), whereas no overall differences between patients and controls were found in the entire hippocampal volume (Fig 1). The analysis of stage-specificity in a 


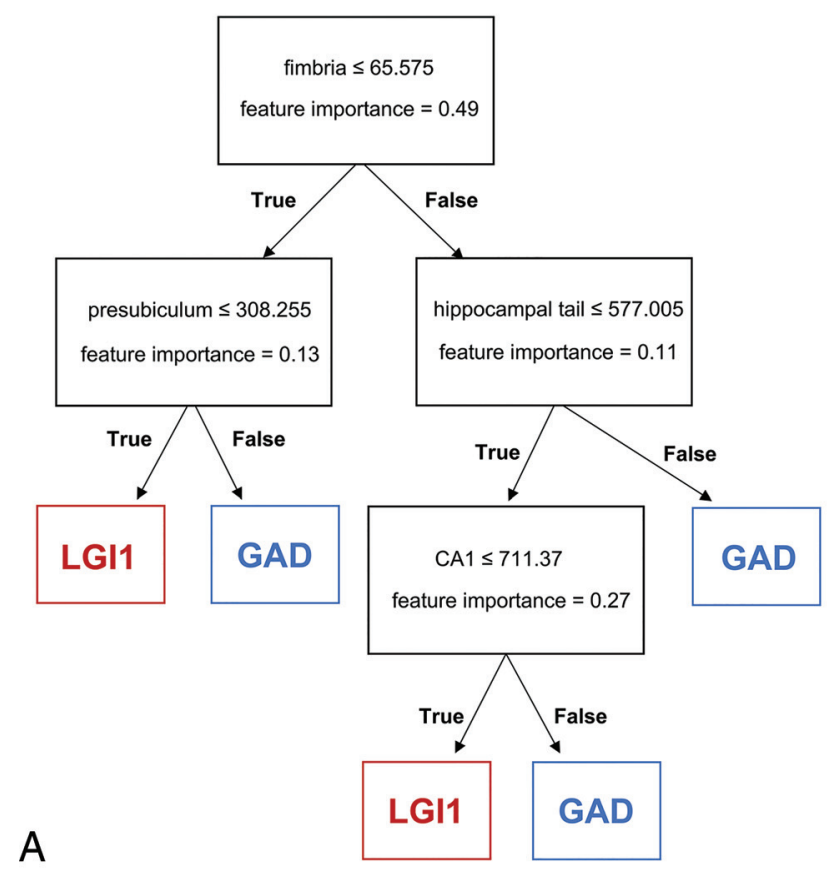

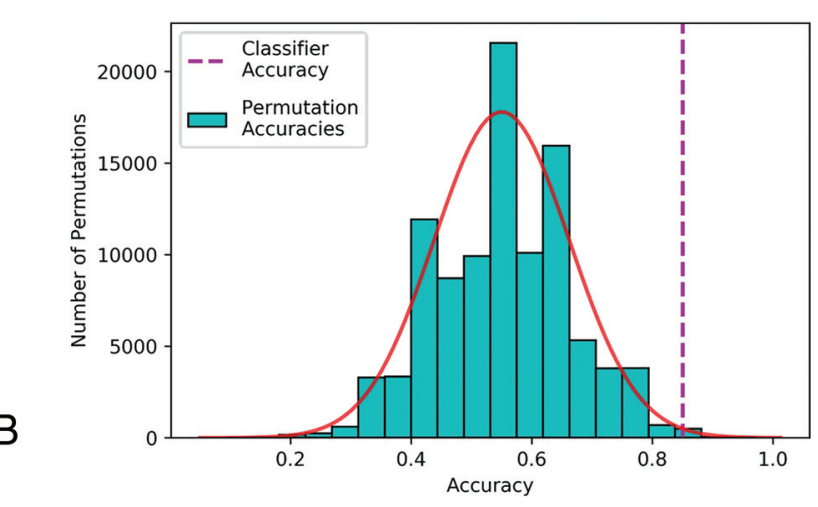

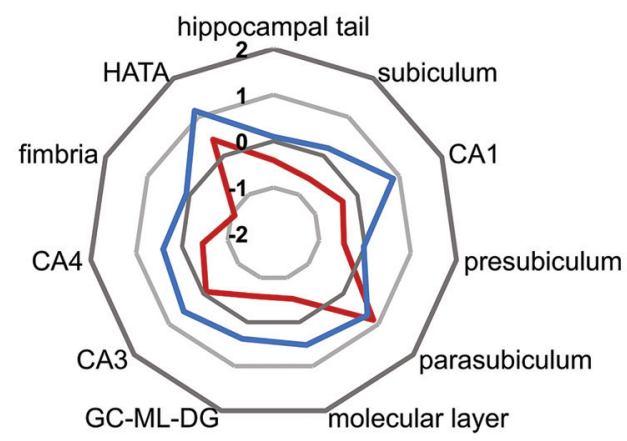

FIG 3. Decision tree classification. Decision tree classification between LGII and GAD using hippocampal subfield volumes from the hemisphere affected in the EEG in early-stage LE. A, Decision tree with importance of features. B. Histogram showing the number of label-shift permutations for each accuracy bin. The solid red line displays a fitted Gaussian curve describing the underlying probability density function. The dashed purple line marks the classifier performance on the real data. C, Starplot showing $Z$ values of the hippocampal subfield volume differences between LGII-LE and GAD-LE in the affected hemispheric side (see supporting information in On-line Tables 3 and 5 for statistics). HATA indicates hippocampus-amygdala transition area; GC-ML-DG, granule cell layer of the dentate gyrus.

retrospective clinical study like the current one is challenged by therapeutic interventions. Whereas most patients in the early group had not undergone immunotherapy, most patients in the late group had (see Table 1 and supporting information in Online Tables 1 and 2). It is, therefore, difficult to determine whether changes observed between the early and the late groups are due to treatment or occur after treatment. Those with GAD-LE have been described as less responsive to immunotherapy compared with those with VGKC-LE, and the conspicuous persistence of mesiotemporal volume alterations in those with late GAD-LE (between-group differences also in late groups) may reflect a poor response to therapy. VGKC-LE, in turn, shows no mesiotemporal volume alterations in the late group, which may be treatment- or disease-related. There has been accruing evidence that abnormal findings on MR imaging are most prominent and specific in the early course of the disease, ${ }^{24,25}$ emphasizing the idea of an MR imaging-based classification on clinical grounds, when early diagnosis is vital for the patient. Higher volumes of mesiotemporal structures in the initial phase of LE may be explained by a functional loss of energy-dependent sodium-potassium adenosine triphosphatase with consecutive cellular swelling and cytotoxic edema due to persistent neuronal damage. ${ }^{26,27}$ The late course of LE, particularly LE with LGI1 autoantibodies, has been reported to result in hippocampal sclerosis as residual damage, characterized by atrophy of the hippocampus. ${ }^{9,25,28}$
Indeed, in our study, LGI1-LE shows the most prominent atrophy of the hippocampus already in an early stage compared with GAD-LE and VGKC-LE, possibly heralding the onset of hippocampal sclerosis (Fig 3C).

\section{Side-Specificity}

Previous MR imaging studies have found biamygdalar swelling in patients with LE. ${ }^{15,25}$ This study is the first to reorient MR imaging volumes according to lateralization of EEG abnormalities, and normal contralateral EEG does not preclude pathologic affection of the contralateral hemisphere. Nevertheless, volume differences between patients and controls after reorientation could be found only in the affected hemispheric amygdala. This suggests that at least in its early phase, LE might be a unihemispheric disease. This notion is corroborated by the unilateral hippocampal signal changes observed in conventional visual assessment in most patients (Table 1). Navarro et $\mathrm{al}^{29}$ have suggested that LGI1-LE may initially evolve in 1 hemisphere and progress to bilateral effects before immunomodulatory treatment. Indeed, in our study, the asymmetry of amygdala volumes was more pronounced in the early group. It is open to discussion whether biamygdalar swelling found in previous studies was reported due to the inclusion of patients with LE evolving in both the left and the right hemisphere or whether it shows the consequence of bihemispheric progression. In any case, there is skepticism as to whether 


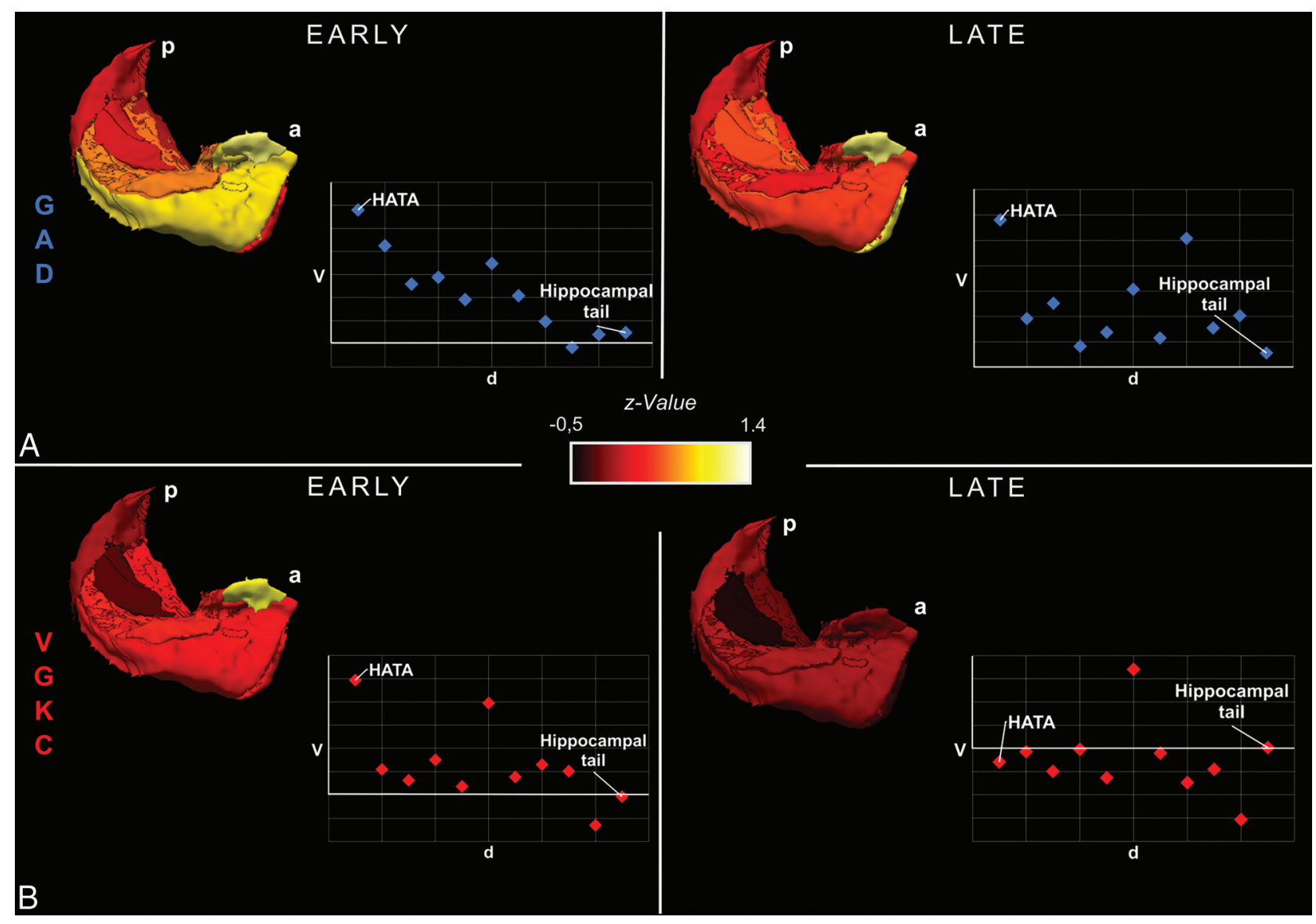

FIG 4. 3D visualization showing hippocampal subfield volume differences between patients and controls. 3D visualization of group differences between all GAD-LE and controls ( $A$ ) as well as between VGKC-LE and controls (B) using hippocampal subfield volumes from the affected hemisphere in early and late stages. Schematic scatterplots show $Z$ values of volumes as a function of the approximate spatial distance from the amygdala. $P$ indicates posterior; a, anterior; $\mathrm{d}$, distance; $\mathrm{V}$, volume. Please note the anterior-to-posterior gradient of subfield volume alterations in early GAD-LE and how the gradient tapers off in late GAD-LE.

bilateral MR imaging abnormalities should be considered a diagnostic criterion in $\mathrm{LE}^{3}$

\section{Autoantibody-Specificity}

The presence of the limbic syndrome and numerous histopathologic studies confirming neuronal loss and lymphocytic infiltration mainly in the amygdala and the hippocampus ${ }^{30,31}$ both lead to the understanding of LE as a mesiotemporal disease. Although autoantibody-specific imaging features have also been found in extratemporal regions, the radiologic MR imaging signature of LE is characterized by hyperintensity in T2WI and/or FLAIR images and volume alteration of the amygdala and hippocampus. ${ }^{12,14}$ In the current study, we observed significantly higher volumes of CA1 and the hippocampus-amygdala transition area on the affected hemisphere in patients with early GAD-LE compared with their matched controls, whereas in early VGKC-LE, this was the case for the hippocampus-amygdala transition area and parasubiculum. Studies in patients with focal lesions limited to the CA1 region of the hippocampus attribute a crucial role in autobiographic memory retrieval, mental time travel, and autonoetic consciousness to CA1. ${ }^{32,33}$ Most interesting, these cognitive functions have been shown to be impaired in GAD-LE, ${ }^{34}$ and the swollen CA1 region in GAD-
LE most likely constitutes the structural correlate of this cognitive deficit.

It is open to discussion why CA1 is selectively affected in GAD-LE. It has been shown that CA1 is particularly vulnerable to a variety of pathologic conditions such as ischemia, inflammation, or increased metabolic demand related to epileptic activity $^{27,35}$ and CA1 neurons express an extraordinary high density of $\mathrm{N}$-methyl-D-aspartate NR2 subunits. ${ }^{36,37}$ Neuronal death in CA1 in GAD-LE may, thus, be caused by a cytotoxic accumulation of $\mathrm{Ca}^{2+}$ following excessive stimulation of the $N$-methyl-Daspartate receptor by an abnormally high accumulation of glutamic acid due to autoantibody-mediated dysfunction of GAD.

A recent study on the structure-function relationship in the healthy human hippocampus suggested an anterior-to-posterior gradient of distinctive temporolimbic connectivity. ${ }^{38}$ The anterior parts of the hippocampus were shown to emphasize associations with memory- and emotion-related terms. This finding is paralleled by the anterior-to-posterior gradient with volume-increased hippocampal regions we found in early GAD-LE and emphasizes its functional relevance (Fig 4). In late GAD-LE, the anterior-toposterior gradient is not prominent, and instead a generalized structural alteration of the hippocampus is observed. It may be most cautiously speculated that this anterior-to-posterior 
gradient in early GAD-LE is indicative of an acute inflammatory process originating from the amygdala and progressing via the hippocampus-amygdala transition area ${ }^{39}$ toward the hippocampal tail along the long hippocampal axis.

\section{Classification}

Markedly, observed mesiotemporal differences were sufficiently defined so that classification was possible on an individual level between LGI1 and GAD by means of supervised machine learning. To the best of our knowledge, this is the first approach of computerized classification between serogroups in LE on the basis of postprocessed MR imaging data described. However, our classification results need to be interpreted cautiously: Despite using a leave-one-out cross-validation scheme to leverage most of our data for training, we cannot rule out possible effects of overfitting due to the low and unbalanced sample size. It has been thoroughly discussed in recent neuroimaging literature that small sample sizes inflate reported accuracies, suggesting that our results are rather over- than underestimating sensitivity and specificity. ${ }^{40}$ Despite these limitations, using permutation testing, we could show that our classifier extracts useful patterns of the hippocampal profiles of the real patient distributions and does not fit random distributions. Nevertheless, due to the described limitations, our findings do not yet translate into gains for patients with LE in more than an experimental setting with a wide range of autoantibody-associated subtypes of LE, or even in those without shown autoantibody association (antibody-negative LE). The recent surge in the discovery of novel LE-associated autoantibodies implies that these patients with antibody-negative LE could quite possibly have yet-unknown autoantibodies. They might obtain a syndrome-based diagnosis only based on their clinical presentation. Thus, it would be very helpful to differentially define imaging correlates of various LE subtypes; however, larger sample sizes are needed for a reliable predictive analysis of possible imaging biomarkers.

\section{CONCLUSIONS}

Diagnostic attention has long been focused on the serostatus of patients with LE. It only shifted to their clinical presentation when the large number of seronegative patients presenting with a limbic syndrome became evident and novel studies bolstered the importance of prompt therapy, even before the results of antibody testing are available. MR imaging is an important pillar of early diagnostics and frequently casts clinical suspicion toward autoimmune encephalitis. However, the MR imaging signature of LE, hallmarked by temporomesial abnormalities, appears to be overly simplistic in light of the current findings and with regard to the wide clinical disease spectrum of LE. The method presented here is clearly not yet applicable in a clinical setting. Instead, it may be seen as a first step toward bridging the gap between a wide clinical disease spectrum and a relatively uniform description of MR imaging findings in patients with LE. Furthermore, our results stress the existence of a multitude of pathologic entities covered by the umbrella term "limbic encephalitis." It is our hope that this study and future imaging research will add weight to using MR imaging within the diagnostic work- up of patients with LE and will contribute to a most detailed description of pathologic entities.

\section{ACKNOWLEDGMENTS}

The authors are grateful for the kind support provided by the Verein zur Förderung der Epilepsieforschung e.V.

Disclosures: Theodor Rüber-RELATED: Grant: grant from BONFOR Research Commission of the Medical Faculty of the University of Bonn. Leon ErnstRELATED: Grant: Promotionskolleg Neuroimmunology of the University of Bonn and the Else-Kröner-Fresenius Stiftung. Christian Elger-UNRELATED: Consultancy: Eisai, Desitin, Novartis, Union Chimique Belge, Medtronic; Employment: Beta Klinik, Bonn; Payment for Lectures Including Service on Speakers Bureaus: Novartis, Cyberonics, Desitin, Eisai; Payment for Development of Educational Presentations: Union Chimique Belge.

\section{REFERENCES}

1. Bien CG, Elger CE. Limbic encephalitis: a cause of temporal lobe epilepsy with onset in adult life. Epilepsy Behav 2007;10:529-38 CrossRef Medline

2. Dalmau J, Graus F. Antibody-mediated encephalitis. $N$ Engl J Med 2018;378:840-51 CrossRef Medline

3. Graus F, Titulaer MJ, Balu R, et al. A clinical approach to diagnosis of autoimmune encephalitis. Lancet Neurol 2016;15:391-404 CrossRef Medline

4. Malter MP, Helmstaedter C, Urbach $\mathrm{H}$, et al. Antibodies to glutamic acid decarboxylase define a form of limbic encephalitis. Ann Neurol 2010;67:470-78 CrossRef Medline

5. Binks SNM, Klein CJ, Waters P, et al. LGI1, CASPR2 and related antibodies: a molecular evolution of the phenotypes. $J$ Neurol Neurosurg Psychiatry 2018;89:526-34 CrossRef Medline

6. Dubey D, Pittock SJ, Kelly CR, et al. Autoimmune encephalitis epidemiology and a comparison to infectious encephalitis. Ann Neurol 2018;83:166-77 CrossRef Medline

7. Van Sonderen A, Ariño H, Petit-Pedrol M, et al. The clinical spectrum of Caspr2 antibody-associated disease. Neurology 2016;87:52158 CrossRef Medline

8. Irani SR, Alexander S, Waters $\mathrm{P}$, et al. Antibodies to Kv1 potassium channel-complex proteins leucine-rich, glioma inactivated 1 protein and contactin-associated protein-2 in limbic encephalitis, Morvan's syndrome and acquired neuromyotonia. Brain 2010;133:2734-48 CrossRef Medline

9. Malter MP, Frisch C, Schoene-Bake JC, et al. Outcome of limbic encephalitis with VGKC-complex antibodies: relation to antigenic specificity. J Neurol 2014;261:1695-705 CrossRef Medline

10. Jayaweera HK, Hickie IB, Duffy SL, et al. Episodic memory in depression: the unique contribution of the anterior caudate and hippocampus. Psychol Med 2016;46:2189-99 CrossRef Medline

11. Finke C, Kopp UA, Scheel M, et al. Functional and structural brain changes in anti-N-methyl-D-aspartate receptor encephalitis. Ann Neurol 2013;74:284-96 CrossRef Medline

12. Wagner J, Schoene-Bake JC, Witt JA, et al. Distinct white matter integrity in glutamic acid decarboxylase and voltage-gated potassium channel-complex antibody-associated limbic encephalitis. Epilepsia 2016;57:475-83 CrossRef Medline

13. Heine J, Prüss $H$, Bartsch $T$, et al. Imaging of autoimmune encephalitis; relevance for clinical practice and hippocampal function. Neuroscience 2015;309:68-83 CrossRef Medline

14. Urbach H, Rauer S, Mader I, et al. Supratentorial white matter blurring associated with voltage-gated potassium channelcomplex limbic encephalitis. Neuroradiology 2015;57:1203-09 CrossRef Medline

15. Wagner J, Weber B, Elger CE. Early and chronic gray matter volume changes in limbic encephalitis revealed by voxel-based morphometry. Epilepsia 2015;56:754-61 CrossRef Medline 
16. Malter MP, Frisch C, Zeitler H, et al. Treatment of immune-mediated temporal lobe epilepsy with GAD antibodies. Seizure 2015;30:57-63 CrossRef Medline

17. Gastaldi M, Thouin A, Vincent A. Antibody-mediated autoimmune encephalopathies and immunotherapies. Neurotherapeutics 2016;13:147-62 CrossRef Medline

18. Fischl B, Salat DH, Busa E, et al. Whole brain segmentation: automated labeling of neuroanatomical structures in the human brain. Neuron 2002;33:341-55 CrossRef Medline

19. Fischl B, Salat DH, Van Der Kouwe AJW, et al. Sequence-independent segmentation of magnetic resonance images. Neuroimage 2004;23(Suppl 1):S69-84 CrossRef Medline

20. Iglesias JE, Augustinack JC, Nguyen K, et al. A computational atlas of the hippocampal formation using ex vivo, ultra-high-resolution MRI: application to adaptive segmentation of in vivo MRI. Neuroimage 2015;115:117-37 CrossRef Medline

21. Milliken GA, Johnson DE. Analysis of Messy Data. London: Chapman \& Hall/CRC; 2009

22. Pedregosa F, Weiss R, Brucher M. Scikit-Learn: machine learning in Python. https://scikit-learn.org/stable/index.html. Accessed February 4, 2019

23. Quinlan JR. Induction of decision trees. Mach Learning 1986;1:81106 CrossRef

24. Finke C, Prüss H, Heine J, et al. Evaluation of cognitive deficits and structural hippocampal damage in encephalitis with leucinerich, glioma-inactivated 1 antibodies. JAMA Neurol 2017;74:50-59 CrossRef Medline

25. Wagner J, Witt JA, Helmstaedter C, et al. Automated volumetry of the mesiotemporal structures in antibody-associated limbic encephalitis. J Neurol Neurosurg Psychiatry 2015;86:735-42 CrossRef Medline

26. Urbach H, Soeder BM, Jeub M, et al. Serial MRI of limbic encephalitis. Neuroradiology 2006;48:380-86 CrossRef Medline

27. Bartsch T, Döhring J, Reuter S, et al. Selective neuronal vulnerability of human hippocampal CA1 neurons: lesion evolution, temporal course, and pattern of hippocampal damage in diffusion-weighted MR imaging. J Cereb Blood Flow Metab 2015;35:1836-45 CrossRef Medline

28. Irani SR, Stagg CJ, Schott JM, et al. Faciobrachial dystonic seizures: the influence of immunotherapy on seizure control and prevention of cognitive impairment in a broadening phenotype. Brain 2013; 136:3151-62 CrossRef Medline
29. Navarro V, Kas A, Apartis E, et al. Motor cortex and hippocampus are the two main cortical targets in LGI1-antibody encephalitis. Brain 2016;139:1079-93 CrossRef Medline

30. Khan NL, Jeffree MA, Good C, et al. Histopathology of VGKC antibody-associated limbic encephalitis. Neurology 2009;72:1703-05 CrossRef Medline

31. Graus F, Saiz A, Lai M, et al. Neuronal surface antigen antibodies in limbic encephalitis: clinical-immunologic associations. Neurology 2008;71:930-36 CrossRef Medline

32. Bartsch T, Alfke K, Stingele R, et al. Selective affection of hippocampal CA-1 neurons in patients with transient global amnesia without long-term sequelae. Brain 2006;129:2874-84 CrossRef Medline

33. Bartsch T, Dohring J, Rohr A, et al. CA1 neurons in the human hippocampus are critical for autobiographical memory, mental time travel, and autonoetic consciousness. Proc Natl Acad Sci U S A 2011;108:17562-67 CrossRef Medline

34. Witt JA, Vogt VL, Widman G, et al. Loss of autonoetic awareness of recent autobiographical episodes and accelerated long-term forgetting in a patient with previously unrecognized glutamic acid decarboxylase antibody related limbic encephalitis. Front Neurol 2015;6:1-8 CrossRef Medline

35. Schmidt-Kastner R, Freund TF. Selective vulnerability of the hippocampus in brain ischemia. Neuroscience 1991;40:599-636 CrossRef Medline

36. Butler TR, Self RL, Smith KJ, et al. Selective vulnerability of hippocampal cornu ammonis 1 pyramidal cells to excitotoxic insult is associated with the expression of polyamine-sensitive N-methyl-dasparate-type glutamate receptors. Neuroscience 2010;165:525-34 CrossRef Medline

37. Lalonde CC, Mielke JG. Selective vulnerability of hippocampal subfields to oxygen-glucose deprivation is a function of animal age. Brain Res 2014;1543:271-79 CrossRef Medline

38. Vos de Wael R, Larivière S, Caldairou B, et al. Anatomical and microstructural determinants of hippocampal subfield functional connectome embedding. Proc Natl Acad Sci USA 2018;115:10154-59 CrossRef Medline

39. Zeidman P, Maguire EA. Anterior hippocampus: the anatomy of perception, imagination and episodic memory. Nat Rev Neurosci 2016;17:173-82 CrossRef Medline

40. Varoquaux G. Cross-validation failure: small sample sizes lead to large error bars. Neuroimage 2018;180:68-77 CrossRef Medline 